\title{
Tunable far infrared laser spectrometers
}

\author{
G. A. Blake, ${ }^{\text {a) }}$ K. B. Laughlin, b) R. C. Cohen, K. L. Busarow, D-H. Gwo, ${ }^{\text {c) }}$ \\ C. A. Schmuttenmaer, D. W. Steyert, and R. J. Saykally \\ Department of Chemistry, University of California, and Materials and Chemical Sciences Division, \\ Lawrence Berkeley Laboratory, Berkeley, California 94720
}

(Received 24 September 1990; accepted for publication 10 March 1991)

The state of the art in far infrared (FIR) spectroscopy is reviewed. The development of tunable, coherent FIR radiation sources is discussed. Applications of tunable FIR laser spectrometers for measurement of rotational spectra and dipole moments of molecular ions and free radicals, vibration-rotation-tunneling (VRT) spectra of weakly bound complexes, and vibration-rotation spectra of linear carbon clusters are presented. A detailed description of the Berkeley tunable FIR laser spectrometers is presented in the following article.

\section{INTRODUCTION}

Defined most conveniently in terms of modern technology, the far infrared (FIR) region of the spectrum extends from the frequencies where "standard" submillimeter techniques begin to fail $\left(\sim 10 \mathrm{~cm}^{-1}=0.3 \mathrm{THz}\right)$ to those where lead-salt infrared diode laser technology becomes operative $\left(\sim 350 \mathrm{~cm}^{-1}=10.5 \mathrm{THz}\right)$. In this frequency range, pure rotational spectra of light molecules and torsional (hindered rotation) spectra of nonrigid molecules have been studied for many years. In terms of wavelength, this spectral region $(30-1000 \mu \mathrm{m})$ is centered near the peak of the room temperature $(3-300 \mathrm{~K})$ blackbody emission spectrum, and has accordingly been of great importance in the development of modern physics. In terms of energy, photons in this region $(0.03-1.0 \mathrm{kcal} / \mathrm{mole})$ are less energetic than essentially all known hydrogen bond (and, of course, chemical bond) strengths, but are about the same magnitude as typical van der Waals bond strengths.

The extremely high resolution $\left(>1 \times 10^{6}\right)$ traditionally associated with microwave spectroscopy has been extended throughout the mid-IR and into the far UV with the continued development and exploitation of modern laser techniques. In contrast, the FIR is still often referred to as "the gap in the electromagnetic spectrum," and remains relatively unexplored with high resolution techniques. Instead, incoherent blackbody sources and dispersive or interferometric spectrometers have traditionally been employed in the FIR, yielding vastly lower sensitivity and resolution than is now routinely achieved in other regions of the spectrum. Exceptions to this scenario are the laser magnetic resonance ${ }^{1,2}$ and laser stark spectroscopy ${ }^{3}$ experiments carried out over the last 15 years with line-tunable FIR gas lasers. While these intracavity resonance experiments have demonstrated extremely high sensitivity and
sub-Doppler resolution, and have certainly produced a considerable number of important results, they are, however, applicable only to paramagnetic or polar molecules, and yield spectra that are more difficult to interpret.

There are numerous systems and phenomena of great current interest in chemistry, physics, and biology, which could be very effectively studied by high resolution spectroscopy in the FIR. These include the determination of intermolecular potential surfaces, studies of hydrogen bond tunneling dynamics, vibrational spectroscopy of metal and covalent clusters, and studies of reactive molecular complexes. In order to address these systems in a general way, tunable coherent sources of FIR radiation are required. In this review article we describe the development of such tunable FIR laser systems and discuss their employment in several of the contexts discussed above. We begin with a brief review of recent technological evolution that has made these new experiments possible.

\section{A BRIEF HISTORY OF SPECTROSCOPY IN THE FAR INFRARED}

\section{A. Broadband spectroscopy}

As was the case for other spectral regions before the invention of lasers, initial work in the FIR was performed with the use of the dispersive elements, such as diffraction gratings, or with Fourier transform spectrometers. Since the throughput of Fourier transform instruments is so large when compared with gratings (no small advantage in a region where simply producing suitably intense radiation is difficult), Fourier transform spectroscopy (FTS) has been considerably more popular for spectral line work. The earliest FTS spectrometers utilized stretched sheets of plastic materials for beamsplitters, which accordingly produce large gaps, or "nulls," in the spectrum. In 1969, Martin and Puplett ${ }^{4}$ proposed and demonstrated a novel polariza-

\footnotetext{
*)Division of Geological and Planetary Science, California Institute of Technology, MS-170-25, Pasadena, CA 91125.

' Research Laboratories, 727 Norristown Road, Rohm \& Haas Company, Spring House, PA 19477.

"Department of Physics, Hansen Laboratory (GP-B, MS-4085), Stanford University, Stanford, CA 94035.
} 
tion interferometer that has found widespread application ever since, especially in our own work. By employing beamsplitters consisting of a finely spaced set of parallel wires to transmit and reflect beams of opposite polarization, interferograms may be collected from nearly dc to frequencies as large as $c / 2 d$, where $d$ is the wire spacing. In addition, sample and background data can be taken simultaneously, thereby producing interferograms which oscillate about the true zero level, allowing complex refractive indices and relative permittivities to be measured across the full FIR with a single device. The most modern polarization FTS instruments use a folded optical configuration to double the effective resolution, which is ultimately limited to about $45-60 \mathrm{MHz}{ }^{5}$ If cooled filters are used to restrict the bandwidths of the detectors, sensitivities as high as $10^{-14} \mathrm{~W} / \mathrm{Hz}^{1 / 2}$ can be reached, enabling some reactive intermediates to be studied.

The principal drawbacks of the Fourier transform technique, as compared to the use of narrowband radiation sources, are the much lower resolution and sensitivity limits obtained. For a single detector covering the entire farinfrared, FTS instruments become background limited unless the optics and chamber are cryogenically cooled. That is, under normal conditions the multiplex advantage of FTS instruments is lost unless narrowband detector arrays are used. To date, however far-infrared detector arrays have not advanced to a sufficient degree to warrant their use. The current state of the art of FIR-FTS is depicted by the work of Johns, ${ }^{6}$ who has measured the absorption spectra of several isotopes of water and many other species from $20-350 \mathrm{~cm}^{-1}$ using a Bomem DA3.002 spectrometer. The resolution of the instrument is $0.004 \mathrm{~cm}^{-1}$ (120 $\mathrm{MHz}$ ) with a frequency accuracy of $0.0002 \mathrm{~cm}^{-1}(6$ $\mathrm{MHz}$ ), using calibration gases such as $\mathrm{HF}, \mathrm{HCl}$, and $\mathrm{H}_{2} \mathrm{O}$. By averaging spectra over limited frequency intervals for the entire hold time of the cryogenic detectors (approximately one full day), a noise level of approximately $1 \%$ is reached. The use of currently available Ge detector arrays could lower this noise limit by a factor of 10, although the minimum attainable linewidth would still remain one or two orders of magnitude broader than the Doppler width of a typical molecule at room temperature.

\section{B. Harmonic generation sources}

The first penetration into the FIR region with coherent sources was achieved by harmonic generation with centimeter wave oscillators in nonlinear point contact diodes, carried out in the early 1950 s at Duke University ${ }^{7}$ Subsequent technical improvements in higher frequency sources, diodes, multiplier housings, and detectors have enabled usable amounts of radiation to be generated up to $1000 \mathrm{GHz}$ in this fashion, ${ }^{8}$ but the optimum sensitivity range is still between 300 and $500 \mathrm{GHz}$. Two types of systems have been developed, namely untuned, broadband multipliers with rather low efficiency, and narrowband, tuned multipliers possessing much higher efficiency. For the broadband devices, the average power lost per harmonic is about $7-8 \mathrm{~dB}$, but this is less for both low harmonics $(n=2,3)$ and for very large harmonic numbers $(n>10)$. For exam- ple, with an input power of $200 \mathrm{~mW}$ from a klystron at 100 $\mathrm{GHz}$, Rothermel, Phillips, and Keene ${ }^{9}$ report the generation of $1 \mathrm{~mW}(200 \mathrm{GHz}), 30 \mu \mathrm{W}(300 \mathrm{GHz}), 1 \mu \mathrm{W}(600$ $\mathrm{GHz})$, and $100 \mathrm{nW}(900 \mathrm{GHz})$ on the various harmonics. For such systems, the crossover frequency for the achievable output power, as compared to typical laser sideband generation spectrometers, is in the range $300-400 \mathrm{GHz}$.

In higher efficiency multipliers, bandwidth is sacrificed for efficiency as tuning is used to optimize a given output frequency. This can be particularly useful for analytical or remote sensing applications where only a few particular fequencies are required. For example, the optimized 350 $\mathrm{GHz}$ triplers offered by Millitech Corporation produce approximately $1.5 \mathrm{~mW}$ over a $50 \mathrm{GHz}$ range with an input power of $40 \mathrm{~mW}$. For operation at higher frequencies, the tripler can be cascaded with a $600 \mathrm{GHz}$ doubler to provide a specified output power of $0.7 \mathrm{~mW}$ over a $100 \mathrm{GHz}$ range with $10 \mathrm{~mW}$ input. Thus, a single tripler-doubler combination would produce $150 \mu \mathrm{W}$ of narrowband radiation at $600 \mathrm{GHz}$. Tunable radiation at frequencies below $650 \mathrm{GHz}$ can thus be produced at higher levels in narrow frequency bands using frequency multiplication methods than can be generated with laser sideband radiation. These sources are difficult to employ in general broadband applications, however.

GaAs diodes have recently been fabricated with $I-V$ curves specifically tailored to generate predominantly a single high-order harmonic with increased efficiency. Selective enhancement of both the ninth harmonic as well as the fifth harmonic have been demonstrated. ${ }^{10}$ This simplifies the harmonic generation process, as stacks of multipliers can be replaced to some extent by the diode fabrication process. The major drawback at the moment is the large parasitic capacitance of the tailored diodes, which limits their use above $300 \mathrm{GHz}$.

\section{Backward wave oscillators and laser diodes}

Spectroscopic methods having potentially high sensitivity throughout the $300-1500 \mathrm{GHz}$ region have been demonstrated by Krupnov and his colleagues in the USSR $^{11}$ and by the spectroscopy group at Lille. ${ }^{12}$ Both groups utilized high frequency backward wave oscillators (BWOs), or carcinotrons, similar to those used in commercial microwave spectrometers. The machining tolerances for these devices are extremely severe, and although they oscillate over wide ranges (e.g., $1200-1500 \mathrm{GHz}$ ), carcinotrons are very expensive and typically have relatively short lifetimes. Moreover, with the recent extension of solid state sources to over $200 \mathrm{GHz}$ (InP Gunn diodes) and the rapid improvement of GaAs Schottky diodes, comparable amounts of power $(50-100 \mu \mathrm{W})$ in the $1 \mathrm{THz}$ region should soon become available from much less expensive, and more durable solid state sources. Harmonic generation for shorter wavelengths will require the development of new, and as yet unknown, materials and devices. A similar situation holds for lead-salt diode lasers, which operate at mid-infrared wavelenghts $\left(350-3000 \mathrm{~cm}^{-1}\right)$. They provide a convenient source of radiation with ample power and versatility for sensitive spectroscopy, but the 
current technology is only capable of producing diodes operating at frequencies down to about $350 \mathrm{~cm}^{-1}(10.5$ $\mathrm{THz}$ ). As such, they have not yet reached into the farinfrared region.

\section{Laser resonance methods}

Gas discharge pumped lasers, such as the $\mathrm{HCN}$ and $\mathrm{H}_{2} \mathrm{O}$ systems, and the more recently developed $\mathrm{CO}_{2}$ optically pumped molecular gas lasers, have been used for quite some time to produce intense line tunable radiation throughout the far infrared. Some efforts have been made to operate FIR lasers at higher pressures, thereby broadening the gain profile and providing some limited tunability (on the order of $100 \mathrm{MHz}$ ), ${ }^{13}$ but in general they are not suitable for broadband spectroscopic applications. For spectroscopy of molecules with either permanent magnetic or electric dipole moments, their eigenstates may be "tuned" by the application of large external magnetic or electric fields into resonance with a fixed laser frequency. Provided the density of lasing transitions is high enough, the spectra of numerous molecules can be recorded with high accuracy once the laser positions are known (several different lines must be used to determine the exact locations of a single eigenstate). Because the molecules themselves are tuned (rather than the laser frequency) and because the experiments can easily be performed inside the laser cavity, the sensitivity of laser magnetic resonance (LMR) and laser electric resonance (LER) spectrometers is extremely high-each is capable of detecting as few as $10^{6}-10^{7} \mathrm{~mol} \mathrm{~cm}^{-3}$. The main drawback of these methods is their limited tuning capabilities, the complexity of the resulting spectra, and the need to extrapolate zero field transition frequencies with complicated model-dependent Hamiltonians from a set of coupled, high field measurements.

Having a great deal of similarity to the well-known ESR technique, LMR has been, by far, the more widely used of these methods. A review of advances in FIR LMR spectroscopy up to 1980 has been presented by Evenson $e t$ al. ${ }^{1}$ with a specific review of LMR of ions given by Saykally. ${ }^{2}$ Since the early work on the $\mathrm{OH}$ and $\mathrm{CH}$ radicals by Evenson et al., ${ }^{1}$ LMR has been extended to the spectra of larger radicals of importance to combustion and atmospheric science $\left(\mathrm{HO}_{2}, \mathrm{CH}_{3} \mathrm{O}\right),{ }^{1}$ to neutral and ionic atomic fine structure transitions relevant to astrochemical studies $\left(\mathrm{C}, \mathrm{O}, \mathrm{C}^{+}, \mathrm{N}^{+}\right),,^{1,2}$ to extremely reactive open shell molecular ions $\left(\mathrm{HF}^{+}, \mathrm{HCl}^{+}, \mathrm{HBr}^{+}, \mathrm{OH}^{+}, \mathrm{H}_{2} \mathrm{O}^{+}\right.$, $\left.\mathrm{H}_{2} \mathrm{~S}^{+}\right){ }^{2}$ to the measurement of radical-radical reaction rates, ${ }^{\prime}$ and most recently, to diatomic metal hydrides $(\mathrm{FeH}, \mathrm{CoH}, \mathrm{NiH}) .^{14,15}$ LMR is applicable only to open shell species, regardless of their charge. FIR LER has been used much less extensively, mostly because the available tuning ranges are so small-especially for asymmetric tops-but it offers the distinct advantage of the capability for studying both open and closed shell species, provided they have a dipole moment. The most recent applications of FIR LER has been to the study of vibrations of the van der Waals bonds in the weakly bound $\mathrm{Ar}-\mathrm{HCl}$ molecule at Berkeley ${ }^{3}$ and at Harvard University. ${ }^{16}$ In these studies, a supersonic free jet expansion inside the laser cavity is used to produce the clusters. These FIR LER spectra of the vibration-rotation eigenstates were used to obtain the intermolecular potential surface of $\mathrm{ArHCl}$ with unprecedented accuracy. ${ }^{17}$

\section{E. Heterodyne spectroscopy and the evolution of tunable coherent FIR radiation sources}

Classical heterodyne emission spectroscopy, developed in the FIR for astronomical observations, typically employs fast mixers $\left(\tau=10^{-12} \mathrm{~s}\right)$ to produce "tunability" around the driving local oscillator (LO) by downconverting observed frequencies near the LO into the microwave region, where signal processing is much simpler. In this case, the tunability is limited by the instantaneous bandwidths of the mixer $(\Delta v=1 / \tau)$ and by the availability of low noise preamplifiers. Generally, for frequencies above $500 \mathrm{GHz}$, these spectrometers have employed GaAs Schottky diode mixers and optically pumped lasers. Atomic and molecular emission lines from the terrestrial atmosphere and from interstellar molecular clouds have been detected up to $3 \mathrm{THz}\left(100 \mathrm{~cm}^{-1}\right)$ with heterodyne receivers. ${ }^{18,19}$ While this approach has obvious appeal for astronomy, it has not yet been fruitfully employed outside of remote sensing applications.

Mixers may also be used in the reverse context, viz. they may also be used as upconverters to generate sidebands of the local oscillator frequency. Two such frequency synthesis techniques have been demonstrated in the past few years for generation of tunable coherent FIR radiation: (1) the use of GaAs mixer diodes to generate tunable sidebands on line tunable FIR molecular gas lasers, ${ }^{20-22}$ and (2) $\mathrm{CO}_{2}$ laser FIR difference frequency generation using metal-insulator-metal (MIM) diodes. ${ }^{23}$ Each method has its own set of advantages and drawbacks relative to the other. Both types of mixer devices have sufficient instantaneous bandwidth to place any desired microwave or millimeter wave frequency onto the carrier radiation, and to respond well at FIR wavelengths. Considering that the MIM diode is also used for frequency mixing in the IR and visible, it is obvously faster than the GaAs diode, but it has a smaller nonlinear impedance characteristic and therefore a poorer conversion efficiency. Accordingly, at frequencies where both technologies work, GaAs diodes produce higher FIR output by several orders of magnitude. This translates directly into spectrometer sensitivity, since detector noise currently limits all operating spectrometers. The major advantage of the MIM diodes are their speed. Because they respond to $\mathrm{CO}_{2}$ laser radiation they may be used to generate radiation at frequencies where FIR lasers currently do not operate conveniently, viz. in the region above $200 \mathrm{~cm}^{-1}$. Also, because $\mathrm{CO}_{2}$ radiation is easily blocked by FIR filters, there is no FIR carrier to reject as when using FIR lasers in frequency mixing applications. Both GaAs and MIM diodes are very fragile, and small transient voltages can easily destroy them.

The first successful results with MIM diode FIR difference frequency lasers were reported by Evenson and 
co-workers ${ }^{23}$ in 1984 , who observed the $\operatorname{CO} J=4 \rightarrow 5$ transition with approximately $200 \mathrm{nW}$ of tunable radiation. In these experiments, one of $\mathrm{CO}_{2}$ lasers, as well as a $\mathrm{CO}_{2}$ reference laser, were frequency stabilized to the Lamb dip of a low pressure $\mathrm{CO}_{2}$ cell. The other mixing laser was a small bore waveguide $\mathrm{CO}_{2}$ laser operating at high pressure, stabilized at a tunable frequency offset from the reference laser. The FIR difference frequency could be tuned \pm 120 $\mathrm{MHz}$ by scanning the waveguide $\mathrm{CO}_{2}$ laser across its pressure broadened gain profile. With the use of $\mathrm{N}_{2} \mathrm{O}$ and isotopically substituted $\mathrm{CO}_{2}$ lasers, the frequency coverage of the FIR with this technique is essentially complete to about $5 \mathrm{THz}$. This is one of the advantages of the Evenson method. High accuracy is the other.

More recently, the MIM diode scheme has been extended to enable third order frequency mixing at a frequency of $v_{\mathrm{CO}_{2}}^{a}-v_{\mathrm{CO}_{2}}^{b} \pm v_{\mathrm{MW}}$ by including a microwave source, at the cost of a factor of at most 2-3 decrease in the tunable FIR (TFIR) power ${ }^{24}$ This eliminates the need to scan either $\mathrm{CO}_{2}$ laser, and increases the tunability substantially. As mentioned, the $\mathrm{CO}_{2}$ laser difference frequency technique has an advantage over optically pumped FIR laser sidebands at high frequencies, because FIR laser lines become rather scarce above approximately $3 \mathrm{THz}$. Moreover, it has the advantage that it is a direct synthesis from accurately frequency and phase locked sources, and the ultimate accuracy is limited primarily by linewidths of the observed transitions and not by spectrometer drift. Indeed, the $\mathrm{CO}_{2}$ laser difference frequency spectrometer ${ }^{25}$ has been used to establish extremely accurate frequency standards in the FIR for the calibration gases $\mathrm{CO}, \mathrm{HF}$, and $\mathrm{HCl}$, which can then be used as secondary standards in a number of other techniques. A variety of transient species have also been detected with this method, as we discuss later.

The first FIR laser sideband results obtained with Schottky diode mixers were presented in a paper by Bi$c^{\prime}$ anić and co-workers ${ }^{20}$ in 1978 . The Nijmegen group at that time had a long history of research with harmonic generation, and was looking for a method that could extend their work to higher frequencies. The FIR laser sideband technique avoids the degradation in performance associated with high frequency harmonic generation by using a FIR laser as a fundamental carrier. Only second-order mixing is therefore required to produce a tunable source in the FIR. Separation of the tunable sidebands from the much more intense fixed frequency carrier is the major difficulty with this technique. The laser source built by Bićanić and co-workers ${ }^{20}$ was an 8 -m-long $\mathrm{HCN}$ laser producing $70 \mathrm{~mW}$ at $891 \mathrm{GHz}$ and $20 \mathrm{~mW}$ at $964 \mathrm{GHz}$. The mixer consisted of a crossed guide multiplier similar to those used for harmonic generation. The microwave and laser power were coupled onto a GaAs Schottky barrier diode via waveguides mounted at right angles to the whisker axis. The sidebands exited via a third waveguide, which launched the tunable FIR beam from an open dish reflector.

A reflection grating monochromator separated the sidebands from the laser carrier, and further rejection of the laser was possible using a metal mesh filster. The op- timum power generated at the sideband frequency was 100 $\mathrm{nW}$, and its frequency spectrum was measured both by using a tunable Fabry-Perot interferometer and by observing pure rotational transitions of $\mathrm{H}_{2} \mathrm{~S}$ and $\mathrm{SO}_{2}$ with amplitude modulation detection.

Subsequent improvements in the Nijmegen spectrometer have included the incorporation of an open structure $\operatorname{mixer}^{26}$ (see below) and higher frequency discharge lasers. ${ }^{27}$ The power levels specified in these later papers has increased to $50 \mu \mathrm{W}$ for the first sideband, with third-order mixing at frequencies $v_{L} \pm 2 v_{\mathrm{MW}}$ yielding $1 \mu \mathrm{W}$ of power. The coverage provided by the $\mathrm{HCN}$ laser extended from 700 to $1200 \mathrm{GHz}$ (including the second-order sideband) and the ultimate sensitivity achieved by their spectrometer in terms of fractional absorption was $1 \times 10^{-5}$.

Shortly after publication of the work of Bićanić and co-workers ${ }^{20}$ Fetterman et $a l_{.}{ }^{21}$ at Lincoln Labs introduced two major advances in the laser sideband technique. By combining an optically pumped FIR laser, which can operate at many different frequencies ( $>2000$, in principle), the technique could be extended to a much wider region of the FIR with much greater spectral coverage. In these experiments, a high-power $\mathrm{CO}_{2}$ laser $(50 \mathrm{~W})$ pumps a vibrational transition in a molecule such as $\mathrm{HCOOH}, \mathrm{CH}_{3} \mathrm{~F}$, $\mathrm{CH}_{3} \mathrm{OH}$, or about sixty other species (including isotopic derivatives), and lasing usually occurs between adjacent rotational levels in the excited state. Strong laser lines have been discovered from $250-6000 \mathrm{GHz}$. Fetterman et al. ${ }^{21}$ quote FIR laser output powers of $30 \mathrm{~mW}$ for laser $\mathrm{HCOOH}$ laser lines at $670,693,716$, and $762 \mathrm{GHz}$. Focusing of the laser radiation onto the GaAs diode whisker antenna, mounted in an open structure corner cube, eventually proved to be much more efficient than the crossed guide multiplier design. In this configuration, for which antenna patterns were first calculated by Krautle, Sauter, and Schultz, ${ }^{28}$ a whisker $4 \lambda$ long is placed symmetrically in a corner reflector, parallel to and $1.2 \lambda$ away from the corner. Fetterman et al. ${ }^{21}$ verified the shape and direction of the antenna pattern using a 100 times scale model.

A four-port Michelson diplexer with a Mylar beamsplitter $^{29}$ served to couple the laser radiation onto the corner cube while simultaneously separating out the sidebands, which were reradiated away from the corner cube in the direction of the incoming laser beam. Using microwaves in the range $2.5-18 \mathrm{GHz}$, coupled onto the diode with coaxial cable, sideband powers of about $100 \mathrm{nW}$ were produced. While used primarily to drive heterodyne mixers for remote sensing applications, this laser sideband source was subesquently used to study the spectroscopy of vibrationally excited $\mathrm{CH}_{3} \mathrm{~F}$ and its energy transfer kinetics upon optical pumping by a pulsed $\mathrm{CO}_{2}$ laser. ${ }^{30}$

More recently, Farhoomand et $a .^{22}$ reported improved performance for a similar laser sideband spectrometer, as well as a more comprehensive characterization of the output power and efficiency. Like Fetterman et al., they employed an optically pumped FIR gas laser and an open structure corner cube mixer. Coupling of laser power into and sideband power out of the mixer was accomplished using the polarizing diplexer first invented for FTS work 
by Martin and Puplett. ${ }^{4}$ The polarizing diplexer can be quickly converted to accommodate a vertical or horizontal laser polarization, a process that was cumbersome with Mylar beamsplitting diplexers. It also has the advantage that polarizers are available that work consistently well at frequencies up to about $100 \mathrm{~cm}^{-1}$, whereas Mylar beamsplitters have a rather narrow frequency range within which the reflectance is close to $50 \%$. For further separation of the sidebands, Farhoomand et al. ${ }^{22}$ used a FabryPerot cavity and also a mesh filter, which could be angle tuned to reject the laser frequency. Using carefully calibrated detectors, they found that $4 \mathrm{~mW}$ of FIR laser power at $693 \mathrm{GHz}$ produced $3.0 \mu \mathrm{W}$ of sideband power, while 22 $\mathrm{mW}$ of $1627 \mathrm{GHz}$ carrier produced $2.5 \mu \mathrm{W}$ of tunable radiation with the same corner cube.

GaAs mixer technology is considerably more mature than that for MIM diodes, and its conversion, noise, and coupling mechanisms are therefore better understood at the present time. Moreover, as molecular beam epitaxial techniques advance, the currently realizable potential for faster, more sensitive GaAs diodes is tremendous, implying that at least an order of magnitude in speed and in conversion efficiency can still be realized, especially once the fabrication of planar devices becomes more routine. Nevertheless, MIM can be also expected to evolve considerably over the next decade.

The current state of the art of MIM-based tunable FIR laser spectrometers is described in recent articles by Fetterman and his co-workers. ${ }^{31}$ Recent descriptions of Schottky diode FIR laser spectrometers have been given by both the Berkeley and Nijmegen groups. In particular, Verhoeve et al. have published a detailed description of the current Nijmegan spectrometer in a recent RSI article. ${ }^{32}$ We present a thorough description of the Berkeley design in the following article. ${ }^{33}$

\section{APPLICATIONS}

The tunable FIR laser spectrometers described in this article have been employed in recent studies of molecular ions, reactive free radicals, and weakly bound molecular clusters. In concluding this review, we describe some of the most interesting results, referring the reader to the orginal literature for details.

\section{A. Rotational spectra and dipole moments of molecular ions}

The use of tunable far-IR lasers for measuring zerofield rotational spectra of molecular ions was pioneered by van den Huevel and Dymanus ${ }^{34}$ in 1982. This group employed a hollow-cathode discharge to generate ions, and published far-IR spectra of $\mathrm{HNN}^{+}, \mathrm{HCO}^{+}, \mathrm{CO}^{+}$, and CF. ${ }^{35}$ This work was subsequently extended to $\mathrm{OH}^{+}$, $\mathrm{OD}^{+}, \mathrm{NH}^{+}$, and $\mathrm{H}_{3} \mathrm{O}^{+}$at Nijmegan. ${ }^{36-39}$

The initial application of the Berkeley FIR spectrometer was also in the study of molecular ions. Rotational transitions in ions generated in a magnetically confined negative glow plasma were detected using either tone burst modulation or second harmonic FM methods. ${ }^{40}$ By measuring the rotational Zeeman splittings of the molecular

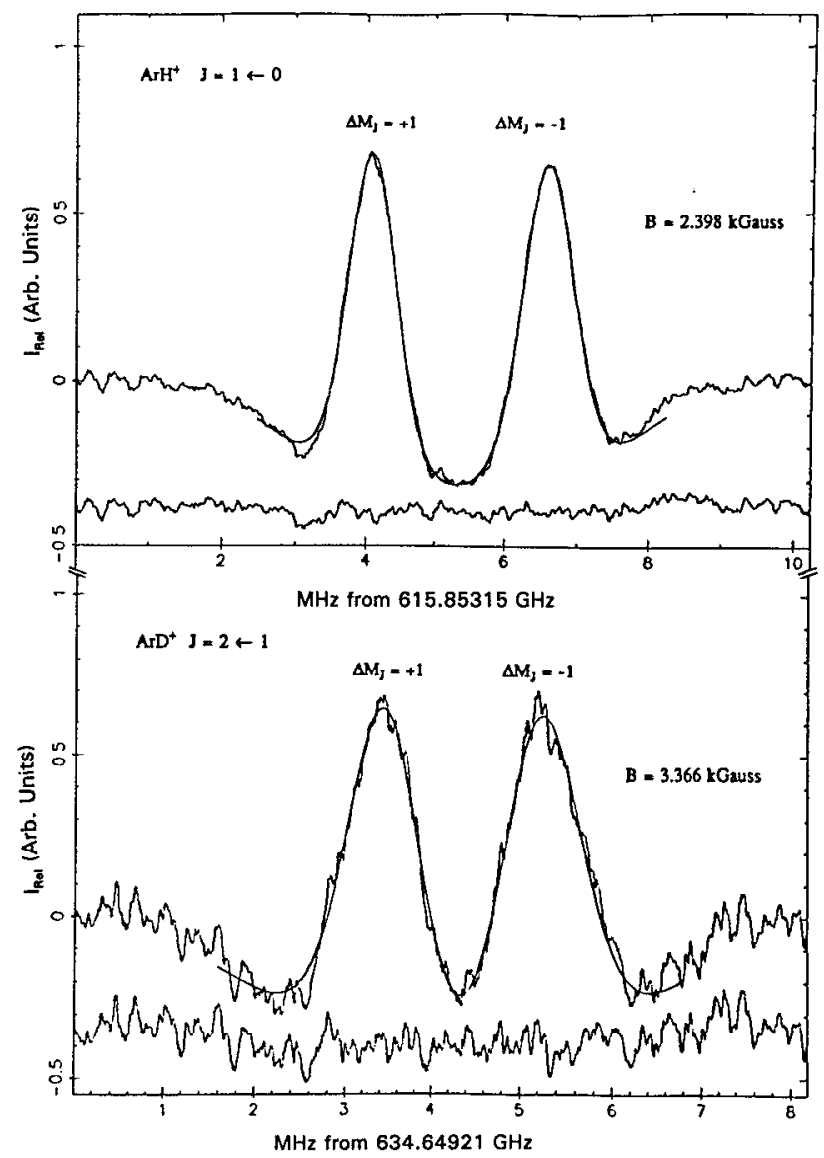

FIG. 1. The rotational Zeeman splitting in the $J=0 \rightarrow 1$ rotational transition of $\mathrm{ArH}^{+}$, observed in a magnetically confined discharge plasma with a tunable FIR laser. This measurement permitted the first experimental determination of the dipole moment of a molcular ion. A general method for extracting permanent dipole moments of ions from such measurements is presented in the papers by Laughlin et al. (Refs. 41-43).

ions in a magnetic field, the first experimental determination of the permanent dipole moment of an ion was carried out by Laughlin et al. in 1987. Representative spectra of the $\mathrm{ArH}^{+}$ion, showing the rotational Zeeman splitting, are shown in Fig. 1. A detailed assessment of this method for determining ion dipole moments has been presented in papers by Laughlin et al. ${ }^{41-43}$ While this initial study was performed using homemade solenoid magnets, similar measurements have recently been carried out at Nijmegen on $\mathrm{HNN}^{+}$by Havenith et al. using a commerical superconducting magnets. ${ }^{44}$ It now appears that tunable FIR laser Zeeman spectroscopy provides a reasonably general method for determining both the magnitudes and signs of molecular ion dipole moments, thus solving a problem that has long eluded spectroscopists.

\section{B. Far infrared vibration-rotation-tunneling (FIR-VRT) spectra of weakly bound complexes}

Perhaps the most powerful experimental configuration yet employed for FIR laser spectrometers involves their use in combination with a continuous planar supersonic jet source, as pioneered by Busarow et al. ${ }^{45,46}$ This experiment has proven to be an important and general new approach for studying intermolecular forces at a greatly enhanced 

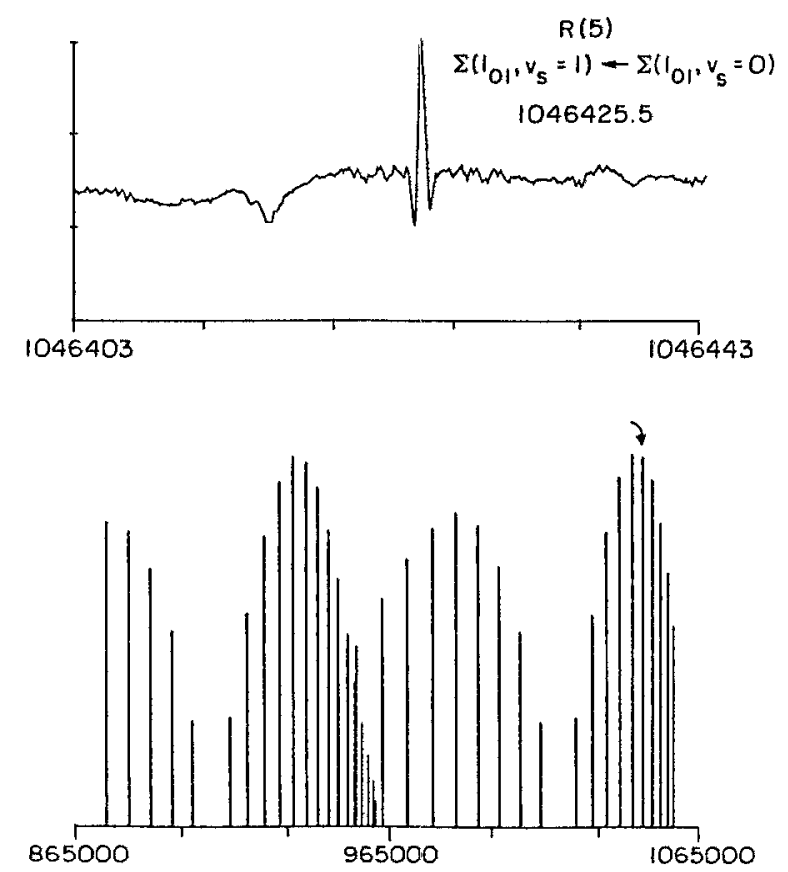

FIG. 2. A vibration-rotation-tunneling (VRT) band of the $\mathrm{Ar}-\mathrm{H}_{2} \mathrm{O}$ van der Waals complex observed in a planar supersonic jet with a tunable FIR laser. The determination of accurate multi-dimensional intermolecular potential energy surfaces (IPS) from FIR-VRT spectra is described in the papers by Cohen et al. (Refs. 47-51).

level of detail, as well as a method for characterizing the complicated many-body tunneling dynamics that occur in hydorgen bonded systems. In the former application, low frequency rovibrational transitions of the weakly bound van der Waals complexes formed and cooled to near $5 \mathrm{~K}$ in the supersonic jet are measured with hyperfine resolution. These data are then inverted with suitable numerical procedures to obtain the intermolecular potential surfacewith far greater accuracy than can be obtained by more

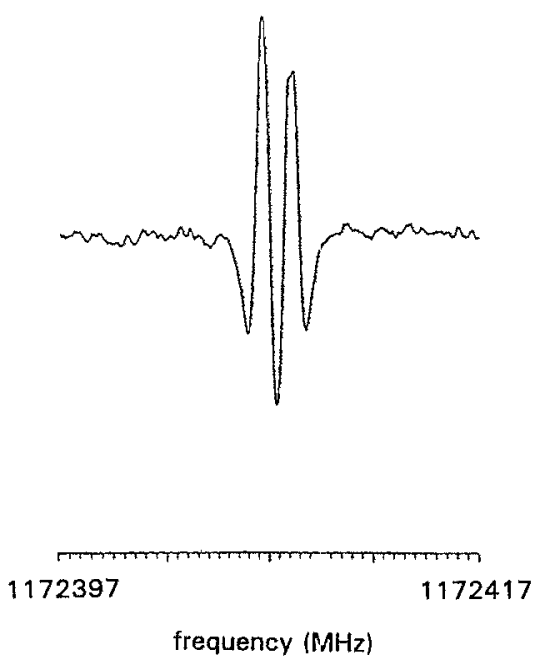

FIG. 3. FIR-VRT spectra of the $\mathrm{Ar}_{2}-\mathrm{HCl}$ cluster. By comparing the IPS determined directly from such measurements with models calculated assuming pairwise additivity, insight is gained concerning the nature of three-body effects in intermolecular forces. See Elrod and co-workers (Ref. 52) for details. conventional methods, such as molecular beam scattering. ${ }^{3}$ A representative scan of such vibration-rotation-tunneling (VRT) transitions in the $\mathrm{Ar}_{2} \mathrm{O}$ complex ${ }^{47-49}$ is shown in Fig. 2. The use of FIR-VRT spectroscopy for the study of intermolecular forces is discussed in detail in two separate reviews. ${ }^{50,51}$ In Fig. 3 are presented representative FIRVRT spectra for the $\mathrm{Ar}_{2} \mathrm{HCl}$ trimer, obtained by Elrod, Steyert, and Saykally. ${ }^{52}$ The goal of this study is to extract an IPS from the FIR data, as for the case of $\mathrm{ArH}_{2} \mathrm{O}$, and to compare it with potentials calculated with the assumption of pairwide additivity. In this manner, one hopes to extract some details regarding the nature of the three-body contributions to intermolecular forces.

A similar application involves measurement of FIRVRT transitions in hydrogen bonded clusters. For example, in the $\mathrm{HCl}$ dimer, ${ }^{53}$ hydrogen exchange dynamics cause the system to tunnel between two equivalent structures (potential minima). The associated tunneling split-
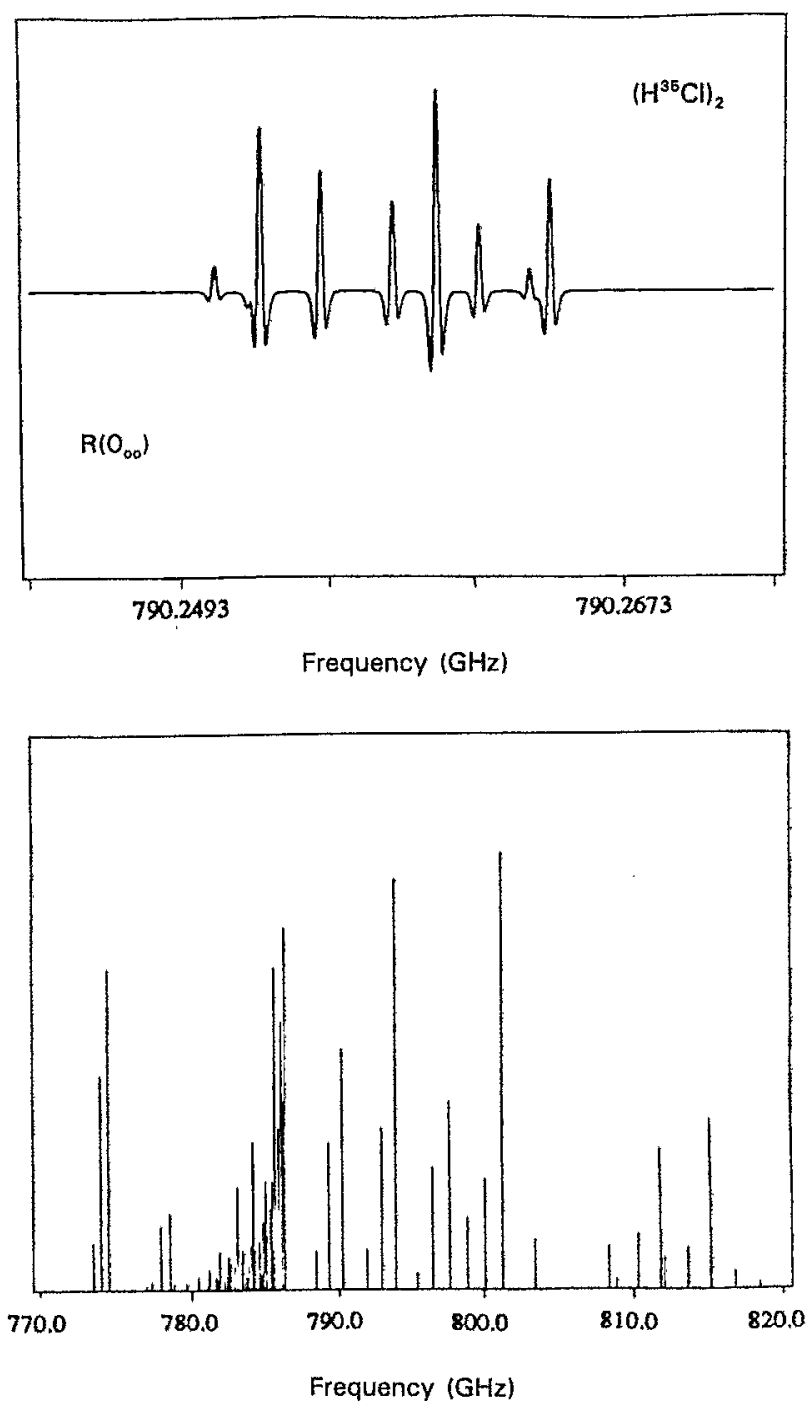

FIG. 4. FIR-VRT spectra of the $\mathrm{HCl}$ dimer, from Blake et al. (Ref. 53) showing the hyperfine structure that results from the quadrupolar $\mathrm{Cl}$ nuclei. Similar spectra have been measured for the water and ammonia dimers, leading to considerable new insight into the hydrogen bond tunneling dynamics that occur in such clusters. 


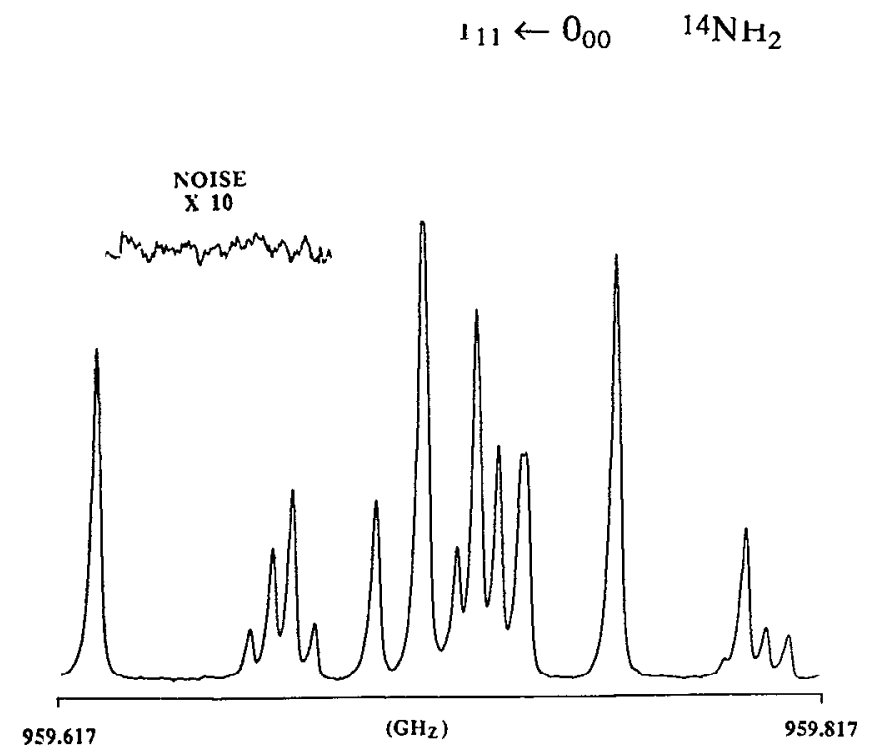

FIG. 5. The $1_{11} \leftarrow 0_{00}$ rotational transition in the ${ }^{2} B_{2}$ ground state of the $\mathrm{NH}_{2}$ radical observed near $30 \mathrm{~cm}^{-1}$ with a tunable FIR laser; ultracold radicals are generated by excimer laser vaporization of a precursor seeded into a planar supersonic expansion, as described by Cohen et al. (Ref. 61).

tings provide a characterization of the potential barrier restricting such motions, as recently demonstrated by Blake et al..$^{54}$ Spectra of the $\mathrm{HCl}$ dimer showing resolution of the nuclear quadrupole hyperfine structure are given in Fig. 4. Analysis of this pattern shows that the two nuclei experience an identical average environment on the time scale of the FIR experiment. Similar measurements have been obtained for $\left(\mathrm{H}_{2} \mathrm{O}\right)_{2}{ }^{55}$ and $\left(\mathrm{NH}_{3}\right)_{2}{ }^{56}$ and $\mathrm{CH}_{4}$ $-\mathrm{H}_{2} \mathrm{O}^{57}$ by the Berkeley group. Meerts and his co-workers have subsequently carried out similar studies on $\left(\mathrm{H}_{2} \mathrm{O}\right)_{2}{ }^{58}$ and $\left(\mathrm{D}_{2} \mathrm{O}\right) .^{59}$ The study of intermolecular forces and hydrogen bond dynamics with FIR-VRT spcctroscopy is becoming a very active field. At least four experimental groups $\mathrm{s}^{50,51,54,58,60}$ are now pursuing such work, and numerous theory groups are working in collaboration.

\section{Ultracold free radicals}

By carrying out UV laser photolysis of suitable precursors in a planar supersonic jet, it has been possible to measure FIR rotational spectra of reactive free radicals. A FIR spectrum of the $\mathrm{NH}_{2}$ radical generated by $193 \mathrm{~nm}$ photolysis of $\mathrm{NH}_{3}$ in an argon expansion is shown in Fig. 5 from the work of Cohen et al. ${ }^{61}$ Similar spectra have been obtained for the $\mathrm{OH}$ and $\mathrm{SH}$ radicals.

\section{Covalent clusters}

By incorporating a Smalley-type supersonic laser vaporization source into the tunable FIR experiment, Schmuttenmaer et $a l .^{62}$ have measured the rovibrational spectrum of the $63 \mathrm{~cm}^{-1}$ bending mode of $\mathrm{C}_{3}$. These spectra, shown in Fig. 6, indicate a rotational temperature near $15 \mathrm{~K}$ for the $\mathrm{C}_{3}$ clusters, and provide the first direct observation of this large amplitude motion. It appears that this

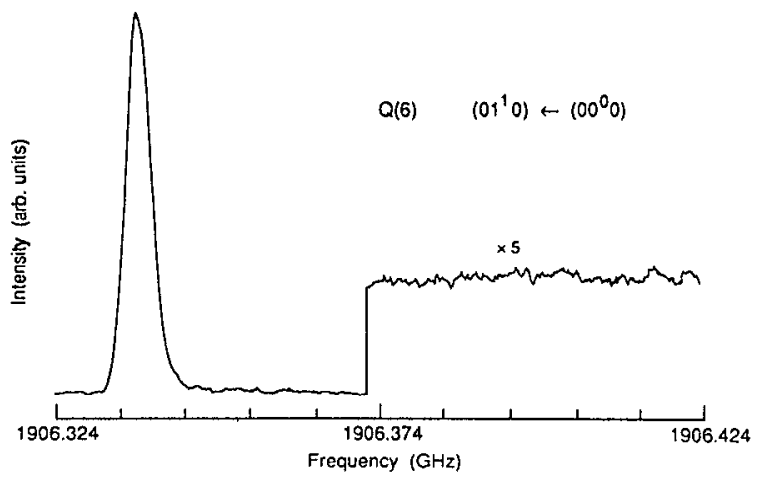

FIG. 6. FIR rovibrational spectra of the $63 \mathrm{~cm}^{-1}$ bending mode of the $C_{3}$ cluster observed by Schmuttenmaer et al. (Ref. 62). Carbon clusters are produced and cooled to near $15 \mathrm{~K}$ by excimer laser vaporization of a graphite target into a supersonic expansion.

method for studying low-frequency vibrations in covalent clusters should be quite general, given the high sensitivity of the method.

\section{DISCUSSION}

It would indeed seem that tunable FIR laser spectroscopy is destined to have an increasingly important role in several different areas of science. There are presently at least six groups (Berkeley, CalTech, JPL, Cambridge, Lille, Nijmegan) operating sideband FIR spectrometers and two others (NIST, Minnesota) operating Fettermantype $\mathrm{CO}_{2}$ laser difference frequency spectrometers. Moreover, several (Berkeley, Caltech, Garching) astronomy groups are specializing in FIR laser hetrodyne methods to study interstellar molecules and star formation processes. While still a technically difficult experiment, tunable farinfrared laser spectroscopy often offers several decided advantages over alternative methods for studying various molecular phenomena. We hope that this review and the detailed description of the Berkeley FIR spectrometer that is presented in the accompanying article will be useful to those scientists who choose to pursue this exciting new avenue of research.

\section{ACKNOWLEDGMENTS}

The Berkeley tunable FIR laser project has been funded by several different agencies over the ten-year development period. These include the National Science Foundation Grant No. CHE-9044712; the Director, Office of Energy Research, Office of Basic Energy Sciences, Chemical Sciences Division of the U.S. Department of Energy under Contract No. DE-AC03-76SF00098; the Army Research Office Grant No. DAAL03-86-G-0184; the Office of Naval Research Grant No. N00014-90-J-1368; and NASA Grant No. NAGW 1022. We thank these agencies for their support.

${ }^{1}$ K. M. Evenson, R. J. Saykally, D. A. Jennings, R. F. Curl, and J. M. Brown, Chemical and Biochemical and Applications of Lasers, edited by C. B. Moore (Academic, New York, 1980), Vol. V.

${ }^{2}$ R. J. Saykally, Chemistry in Britain, February 1985, pp. 159-161.

${ }^{3}$ R. J. Saykally, Accts. Chem. Res. 22, 295 (1989). 
${ }^{4}$ D. H. Martin and E. Puplett, Infra, Phys. 10, 105 (1969).

${ }^{5}$ I. G. Nolt, S. Predko, J. V. Radostitz, J. T. Moseley, P. A. R. Ade, F. D: Robinson, and K. V. Chance, 11th Int. Conf. IR and MM Waves (1986).

${ }^{6}$ J. W. C. Johns, J. Opt. Soc. Soc. Am. B 2, 1340 (1985).

${ }^{7}$ W. Gordy, Proc. Symp. MM Waves, Poly. Press, Poly. Inst. of Brooklyn, NY (1960).

${ }^{8}$ P. Helminger, J. K. Messer, and F. C. DeLucia, Appl. Phys. Lett. 42, 309 (1983).

${ }^{9} \mathrm{H}$. Rothermel, T. G. Phillips, and J. Keene, Int. J. Infr. Millimeter Waves 10, 83 (1989).

${ }^{10}$ Elliot Brown (private communication, 1988)

"A. F. Krupnov, Modern Aspect of Microwave Spectroscopy, edited by G. W. Chantry, 217 (1979).

${ }^{12}$ M. Bogey, C. Demuynck, M. Denis, and J. L. Destombes, Astron. Appl. 148, L11 (1985).

${ }^{13}$ H. O. Everitt, D. D. Skatrud, and F. C. DeLucia, Appl. Phys. Lett. 49, 995 (1986).

${ }^{14}$ S. P. Beaton, K. M. Evenson, T. Nelis, and J. M. Brown, J. Chem. Phys. 89, 4446 (1988).

${ }^{15}$ K. M. Evenson, R. J. Saykally, D. A. Jennings, R. F. Curl, Jr., and J M. Brown, Proc. Yamada Conf. III on Free Radicals, edited by $\mathrm{Y}$ Moriana, I. Tanaka, E. Hirota, K. Obi, and S. Saito (Association for Science Documents, 1979), p. 159.

${ }^{16}$ M. D. Marshall, A. Charo, H. O. Leung, and W. Klemperer, J. Chem. Phys. 83, 4924 (1985).

${ }^{17}$ J. M. Hutson, J. Chem. Phys. 89, 4550 (1988).

${ }^{18}$ R. T. Boreiko, A. L. Betz, and J. Zmuidzinas, Astrophys. J. 325, L47 (1988).

${ }^{19}$ R. T. Boreiko. A. L. Betz, and J. Zmuidzinas, Astrophys. J. 353, 181 (1990).

${ }^{20}$ D. D. Bicanic, B. F. J. Zuidberg, and A. Dymanus, Appl. Phys. Lett. 32, 367 (1978).

${ }^{21}$ H. R. Fetterman, P. E. Tannenwald, B. J. Clifton, W. D. Fitzgerald, and N. R. Erickson, Appl. Phys. Lett. 33, 151 (1978).

${ }^{22}$ J. Farhoomand, G. A. Blake, M. A. Frerking, and H. M. Pickett, J Appl. Phys. 57, 1763 (1985).

${ }^{23}$ K. M. Evenson, D. A. Jennings, and F. R. Peterson, Appl. Phys. Lett. 44. 576 (1984).

${ }^{24}$ K. M. Evenson, D. A. Jennings, K. R. Leopold, and L. R. Zink, Laser Spectrascopy VII, Papers from the Seventh International Conference on Laser Spectroscopy, edited by T. W. Hansch and Y. R. Shen (Springer, Berlin, 1985), p. 300

${ }^{25}$ I. G. Nolt, J. V. Radostitz, G. Dilonardo, K. M. Evenson, D. A. Jennings, K. R. Leopold, M. D. Vanek, L. R. Zink, A. Hinz, and K. V. Chance, J. Mol. Spectrosc. 125, 274 (1987).

${ }^{26}$ F. C. van den Heuvei, Ph.D. Thesis, Katholieke Universiteit, Nijmegen (1982).

${ }^{27}$ F. C. van den Heuvel and A. Dymanus, Chem. Phys. Lett. 92, 219 (1982).

${ }^{28}$ H. Krautle, E. Sauter, and G. V. Schultz, Infrared. Phys. 17, 477 (1977).

${ }^{29}$ N. R. Erickson, IEEE Trans. Microwave Theory Tech, 25, 865 (1977).

${ }^{30}$ W. A. M. Blumberg, H. R. Fetterman, D. D. Peck, and P. F. Goldsmith, Appl. Phys. Lett. 35, 582 (1979).

${ }^{31}$ L. R. Zink, K. M. Evenson, F, Matusushima, T. Neilis, and R. Robinson, Astrophys. J. 371, (1991); K. M. Fetterman, D. A. Jennings, and M. C. Vanek, in Proceedings NATO School on Frontiers of Laser Spectroscopy, edited by A. C. P. Alves (Kluwer Academia, 1988), pp. 43-51.
${ }^{32}$ P. Verhoeve, E. Zwart, M. Versiuis, J. ter Meulen, W. L. Meerts, A. Dymanus, and D. McLay, Rev. Sci. Instrum. 61, 1612 (1990).

${ }^{33}$ G. A. Blake, K. B. Laughlin, R. C. Cohen, K. L. Busarow, D-H. Gwo, C. A. Schmuttenmaer, D. W. Steyert, and R. J. Saykalley, Rev, Sci. Instrum. 62, 1701 (1991).

${ }^{34}$ F. C. van den Heuvel and A. Dynamus, Chem. Phys. Lett. 92, 219 (1982).

${ }^{35}$ F. C. van den Heuvel, W. L. Meerts, and A. Dymanus, Chem. Phys. Lett. 88, 59 (1982).

${ }^{36}$ J. P. Bekooy, P. Verhoeve, W. L. Meerts, and A. Dymanus, J. Chem. Phys. 82, 3868 (1985).

${ }^{37}$ P. Verhoeve, J. P. Bekooy, W. L. Meerts, J. J. ter Meulen, and A Dymanus, Chem. Phys. Lett. 125, 286 (1986).

${ }^{38}$ P. Verhoeve, J. J. ter Meulen, W. L. Meerts, and A. Dymanus, Chem. Phys. Lett. 132, 213 (1986).

${ }^{39}$ P. Verhoeve, J. J. ter Meulen, W. L. Meerts, and A. Dymanus, Chem. Phys. Lett. 143, 501 (1988).

${ }^{40}$ G. A. Blake, K. B. Laughlin, R. C. Cohen, K. L. Busarow, and R. J. Saykally, Astrophys. Lett. 316, L45 (1987).

${ }^{41}$ K. B. Laughlin, G. A. Blake, R. C. Cohen, D. C. Hovde, and R. J. Saykally, Phys. Rev. Lett. 58, 996 (1987).

${ }^{42}$ K. B. Laughlin, G. A. Blake, R. C. Cohen, D. C. Hovde, and R. J. Saykally, Philos. Trans. R. Soc. (London) A 324, 109 (1987).

${ }^{43}$ K. B. Laughlin, G. A. Blake, R. C. Cohen, and R. J. Saykally, J. Chem. Phys. 90, 1358 (1989).

${ }^{44}$ M. Havenith, E. Zwart, W. L. Meerts, and J. J. ter Meulen, J. Chem. Phys, 93, 8446 (1990).

${ }^{45}$ K. L. Busarow, G. A. Blake, K. B. Laughlin, R. C. Cohen, Y. T. Lee, and R. J. Saykally, Chem. Phys. Lett. 141, 289 (1987).

${ }^{46}$ K. L. Busarow, G. A. Blake, K. B. Laughlin, R. C. Cohen, Y. T. Lee, and R. J. Saykally, J. Chem. Phys. 89, 1268 (1988).

${ }^{47}$ R. C. Cohen, K. L. Busarow, K. B. Laughlin, G. A. Blake, M. Havenith, Y. T. Lee, and R. J. Saykally, J. Chem. Phys. 89, 4944 (1988).

${ }^{48}$ R. C. Cohen, K. L. Busarow, Y. T. Lee, and R. J. Saykally, J. Chem. Phys. 92, 169 (1990)

${ }^{49}$ R. C. Cohen and R. J. Saykally, J. Phys. Chem. 94, 7991 (1990).

${ }^{50}$ R. C. Cohen and R. J. Saykally, Ann. Rev. Phys. Chem. 42 (1991).

${ }^{51}$ R. C. Cohen and R. J. Saykally, J. Phys. Chem. (Invited Review) (to be published).

${ }^{52}$ M. J. Elrod, D. W. Steyert, and R. J. Saykally, J. Chem. Phys. 94, 58 (1991).

${ }^{53}$ G. A. Blake, K. L. Busarow, R. C. Cohen, K. B. Laughin, Y. T. Lee, and R. J. Saykally, J. Chem. Phys. 89, 6577 (1988).

${ }^{54}$ G. A. Blake and R. E. Bumgarner, J. Chem. Phys. 91, 7300 (1989).

${ }^{55}$ K. L. Busarow, R. C. Cohen, G. A. Blake, K. B. Laughlin, Y. T. Lee, and R. J. Saykally, J. Chem. Phys. 90, 3937 (1989).

${ }^{56}$ M. Havenith, R. C. Cohen, K. L. Busarow, D. H. Gwo, Y. T. Lee, and R. J. Saykally, J. Chem. Phys. 94, 4776 (1991).

${ }^{57}$ R. C. Cohen, C. A. Schmuttenmaer, K. L. Busarow, and R. J. Saykally, J. Chem. Phys. (to be published).

${ }^{58}$ E. Zwart, J. J. ter Meulen, and W. L. Meerts, in Submillimeter Spectroscopy of Molecular Complexes and Ions (Haveka B. V., Amsterdam, The Netherlands, 1963), p. 38.

${ }^{59}$ Ibid., p. 53. 61

${ }^{60}$ D. W. Firth, M. A. Dvoskak, S. W. Reeve, R. S. Ford, and K. R. Leopold, Chem. Phys. Lett. 168, 161 (1990).

${ }^{61}$ R. C. Cohen, K. L. Busarow, C. A. Schmuttenmaer, Y. T. Lee, and R. J. Saykally, Chem. Phys. Lett. 164, 321 (1989).

${ }^{62}$ C. A. Schmuttenmaer, R. C. Cohen, N. Pugliano, J. R. Heath, A. L. Cooksy, K. L. Busarow, and R. J. Saykally, Science 249, 897 (1990).

\section{PRICING AND ORDERING INFORMATION FOR REVIEW-ARTICLE REPRINTS}

PRICES: $\quad \$ 5.00 ; \$ 4.50$ each for bulk orders of ten or more copies of the same article sent to one address. Delivery is via surface mail Airmail delivery available at the following surcharge: $\$ 2.50$ for the first copy plus $\$ 1.00$ for each additional copy sent to one address. Reprint orders must be prepaid.

ORDERS: Please specify REVIEW OF SCIENTIFIC INSTRUMENTS REVIEWS and give the article title, authors, month, and year of publication, and the page number of the article's title page. Send orders accompanied by payment in full (make checks payable to American Institute of Physics) to: Current Physics Reprints, American Institute of Physics, 335 East 45th Street, New York, NY 100I7. 